\title{
The role of M-mode echocardiography in patients with heart failure and preserved ejection fraction: A prospective cohort study
}

\author{
XIN LI and XINWEN MIN \\ Department of Cardiovascular Medicine, Cardiovascular Research Institute, \\ Affiliated Dongfeng Hospital, Hubei University of Medicine, Shiyan, Hubei 442008, P.R. China
}

Received October 13, 2018; Accepted November 14, 2019

DOI: $10.3892 /$ etm.2020.8428

\begin{abstract}
Epicardial movement during diastole is inversely proportional to myocardial stiffness but systolic regional thickening cannot precisely identify ischemic territories. The aim of the present study was to test the hypothesis that a correlation may be present between M-mode echocardiography parameters and poor outcomes in patients with heart failure and preserved ejection fraction. Patients with known cardiovascular disease were included in the test group $(n=1,244)$ and patients without known cardiovascular disease were included in the control group $(n=1,952)$. Patient records of routine measurements, M-mode echocardiography and mortality were collected. The control population and test population had the same left ventricular end-diastolic dimension $(\mathrm{P}=0.062)$ and left ventricular end-diastolic volume $(\mathrm{P}=0.053)$. A lower mitral flow velocity $(\mathrm{P}<0.05)$, higher Tei index $(\mathrm{P}<0.0001)$ and reduced distribution of diastolic wall strain $(\mathrm{P}<0.0001)$ were reported in the test populations compared with the control population. Patients of the test population with lower diastolic wall strain $(<0.28)$ demonstrated a higher mortality rate than those with higher diastolic wall strain $(\geq 0.28$; $\mathrm{P}<0.0001)$ at the 3-year follow-up. M-mode echocardiographic parameters may be of use for predicting poor outcomes in patients with heart failure and preserved ejection fraction.
\end{abstract}

Correspondence to: $\mathrm{Dr}$ Xinwen Min, Department of Cardiovascular Medicine, Cardiovascular Research Institute, Affiliated Dongfeng Hospital, Hubei University of Medicine, 16 Daling Road, Shiyan, Hubei 442008, P.R. China

E-mail: mathiesenjondee@yahoo.com

Abbreviations: DWS, diastolic wall strain; STARD, standards for the reporting of diagnostic accuracy studies; STROBE, the strengthening the reporting of observational studies in epidemiology; LDL, low-density lipoprotein; HDL, high-density lipoprotein; BNP, brain natriuretic peptide; SF, shortening fraction; DICOM, digital imaging and communications in medicine; CMR, cardiac magnetic resonance; HbAlc, glycated hemoglobin

Key words: diastolic wall strain, ejection fraction, heart failure, mitral flow velocity, M-mode echocardiography

\section{Introduction}

Most patients with heart failure have a preserved ejection fraction (1). In cases of heart failure, population with preserved ejection fraction is associated with higher mortality rates compared with that of healthy hearts (2). Ethnicity has a major impact on the mortality and morbidity of patients with heart failure and preserved ejection fraction $(2,3)$. Asian patients with heart failure and preserved ejection fractions have poorer outcomes compared with Caucasian patients $(2,4,5)$.

The pathophysiology behind heart failure with preserved ejection fraction is complicated and therefore difficult to diagnose (6). However, diastolic stiffness (which presents myocardial relaxation and filing pressure) of the left ventricle serves a crucial role in the pathophysiology (7). Diastolic stiffness is measured using M-mode echocardiography. According to the linear elastic theory, the diastolic epicardial movement is a noninvasive measurement of the left ventricular wall distensibility (8). Echocardiography is able to provide a comprehensive assessment of the anatomy and physiology of the heart (9). Echocardiographic parameters of anatomical and physiological changes of the heart provide a framework for providing effective prognoses and outcomes in the management of patients with heart failure and preserved ejection fraction (10).

One of the echocardiographic parameters, the diastolic wall strain (DWS), is a physical property of the myocardial tissue. There is less myocardial thinning and less wall movement in patients with heart failure and preserved ejection fraction compared with cardiac healthy patients (8). DWS predicts the presence of heart failure with preserved diastolic function (7). An experimental study demonstrated that the epicardial movement during diastole is inversely proportional to the myocardial stiffness of heart failure in rats with preserved ejection fraction (8). Until recently, DWS has not been examined in heart failure patients with preserved ejection fraction.

The primary aim of the present study was to investigate the relationship between M-mode echocardiographic parameters and the structure and function of the heart. The secondary endpoint of the workup was to test the hypothesis that a correlation is present between M-mode echocardiography parameters and poor outcomes of patients with heart failure and preserved ejection fraction. The tertiary aim of the present study was to identify an independent parameter associated with mortality 
of patients with heart failure and preserved ejection fraction in Chinese populations.

\section{Materials and methods}

Inclusion criteria. Patients with heart failure and symptoms including left ventricular dysfunction, palm edema, plasma brain natriuretic peptide $(\mathrm{BNP}) \geq 100 \mathrm{pg} / \mathrm{ml}$, pulmonary capillary wedge pressure $\geq 15 \mathrm{mmHg}$ and/or left ventricular end-diastolic pressure at rest $\geq 12 \mathrm{mmHg}$, with an ejection fraction (measured by echocardiography) $>50 \%$ and no pericardial disease, hypertrophic/infiltrative cardiomyopathy or significant left-sided valvular disease, were included in the test populations of the current study. Patients without known cardiovascular disease, hypertension, obesity (according to body mass index) or diabetes (according to blood glucose levels) were included in the control population of the current study (Table I).

Exclusion criteria. Patients aged $<45$ years for the test population, $<65$ years for the control population and those that had not signed an informed consent form were excluded from the present study. Patients with heart failure and an ejection fraction $<50 \%$, posterior wall motion abnormalities, hypertrophic cardiomyopathy, pericardial effusion, infiltrative cardiomyopathy and significant left-sided valvular disease were excluded from the test population of the current study. Patients who displayed heart failure risk factors (such as cardiovascular disease, hypertension, obesity and diabetes) were excluded from the control population of the current study. Patients who presented ambiguous images that were difficult to interpret and draw conclusions from during diagnosis were excluded from analysis.

Cohorts. Patients with known cardiovascular disease were included in the test group $(n=1,244)$ and patients without known cardiovascular disease were included in the control group $(\mathrm{n}=1,952)$.

Routine measurements. Blood pressure was measured using a sphygmomanometer (Omron HEM-8712; Omron Healthcare, Inc.). Hypertension was defined as systolic blood pressure and diastolic blood pressure $>140$ and $>90 \mathrm{mmHg}$, respectively (11). Blood samples were collected from all enrolled patients and samples were evaluated for total cholesterol, low-density lipoprotein (LDL), high-density lipoprotein (HDL), blood serum creatinine, random blood plasma glucose levels, hemoglobin, and \% glycated hemoglobin (\% HbA1c, normal value: $57 \%$ ) (12). Patients were considered diabetic if random plasma glucose was $>140 \mathrm{mg} / \mathrm{dl}$. Hyperlipidemia was defined as a total cholesterol level $>240 \mathrm{mg} / \mathrm{dl}$, LDL $>160 \mathrm{mg} / \mathrm{dl}$ or/and HDL $<40 \mathrm{mg} / \mathrm{dl}$ (11). ELISA kits (NPPB/BNP Human ELISA Kit; cat. no. EHNPPB; Thermo Fisher Scientific, Inc.) were used to determine BNP (13).

M-mode echocardiography. Ultrasound equipment (iE33; Philips Medical Systems, Inc.) was used to perform M-mode echocardiography using cardiac ultrasonographers. The dimensions, shortening fraction (SF) and the left ventricle's wall thickness was measured from the long-axis view using a 2-3 MHz transducer (14).

DWS was calculated using the following equation (8): DWS $=($ left ventricle posterior wall thickness at end systole-left ventricle posterior wall thickness at end diastole)/left ventricle posterior wall thickness at end diastole. Echo machine data were printed out, magnified to $300 \%$, and measurements were performed manually.

A four-chambered view was taken for the mitral flow velocity and the myocardial wall motion velocity measurements. The sample was placed at the mitral leaflet's tip. Peak atrial and the peak early diastolic flow velocities were determined. The mitral flow E/A was calculated using the following equation (8): Mitral flow $(\mathrm{E} / \mathrm{A})=$ (peak early diastolic flow velocity)/(peak atrial flow velocity). The sample was kept at the lateral site of an atrial-ventricular valve annulus. The peak systolic velocity and the peak early diastolic wall velocity was measured. E/e' ratio was measured using the following equation (8): E/e' ratio=peak early diastolic flow velocity/peak early diastolic wall velocity.

The Tei index was calculated using the following equation: Tei index $=(X-Y) / Y$. Where $X$ represents the time between the mitral annular late diastolic wave trailing edge and the subsequent mitral annular early diastolic wave leading edge. $\mathrm{Y}$ represents the time between the leading edge of the mitral annular systolic wave and the trailing edge of the mitral annular systolic wave (15).

Mortality records were collected from Digital Imaging and Communications in Medicine $\left(\right.$ DICOM $\left.^{\circledR}\right)$.

Statistical analysis. For all parameters of M-mode echocardiography, the mean value of five individual heartbeats were considered for statistical analysis. Individual heartbeat was measured at the time interval of $5 \mathrm{~min}$ of the rest period. Categorical data were analyzed using the Mann-Whitney U test (7) and continuous data were analyzed using unpaired t-test (16). Bland-Altman analysis was used to check intra-observer and inter-observer reliabilities (14). Multiple regression analysis was used to analyze survival rates. Statistical analysis was performed using InStat (Windows version 3.01; GraphPad Software, Inc.). P<0.05 was considered to indicate a statistically significant difference.

\section{Results}

Demographic characters and medical history. A total of 1,556 patients with heart failure were admitted to the Affiliated Dongfeng Hospital, China between 15th August 2012 and 1st August 2015. Among these, there were a total of 145 patients $<45$ years, images of 7 patients were indicated to be inadequate, 71 patients had ejection fraction $<50 \%, 17$ patients had posterior wall motion abnormalities, 19 patients had hypertrophic cardiomyopathy, 15 patients had pericardial effusion, 11 patients had infiltrative cardiomyopathy and 27 patients had left-sided valvular disease; therefore, these patients were excluded from the test population. From the Affiliated Dongfeng Hospital, China and the same referring hospitals of China during the same time period, a total of 2,286 patients were available at an outpatient setting without known cardiovascular disease. 
Table I. Demographics and social characteristics of enrolled patients.

\begin{tabular}{|c|c|c|c|}
\hline \multirow[b]{2}{*}{ Characteristics } & \multicolumn{2}{|c|}{ Group } & \multirow{2}{*}{$\begin{array}{c}\text { Comparison between } \\
\text { groups, P-value }\end{array}$} \\
\hline & Test population & Control population & \\
\hline Number of patients enrolled & 1,244 & 1,952 & $\mathrm{~N} / \mathrm{A}$ \\
\hline \multicolumn{4}{|l|}{ Age, years } \\
\hline Minimum & 45 & 65 & \\
\hline Maximum & 90 & 90 & \\
\hline Mean \pm SD & $61.12 \pm 4.12$ & $71.75 \pm 11.15$ & $<0.0001$ \\
\hline \multicolumn{4}{|l|}{ Sex } \\
\hline Male & $807(65)$ & $1,326(68)$ & \\
\hline Female & $437(35)$ & $626(32)$ & 0.079 \\
\hline Height, $\mathrm{m}$ & $1.56 \pm 0.09$ & $1.55 \pm 0.17$ & 0.056 \\
\hline Smokers & $187(15)$ & $343(18)$ & 0.066 \\
\hline Blood serum creatinine & $1.20 \pm 0.2$ & $1.21 \pm 0.05$ & 0.035 \\
\hline Waist circumference ${ }^{a}$ & $95.12 \pm 5.27$ & $89.56 \pm 7.51$ & $<0.0001$ \\
\hline Routine exercise & Tai Chi & $\begin{array}{l}\text { No exercise except } \\
\text { occasional walking }\end{array}$ & N/A \\
\hline Alcohol intake & $115(9)$ & $85(4)$ & $<0.0001$ \\
\hline Live steatosis ${ }^{\mathrm{b}}$ & $21(2)$ & $0(0)$ & $<0.0001$ \\
\hline \multicolumn{4}{|l|}{ Types of heart failure } \\
\hline Right-sided & $545(43)$ & $0(0)$ & \\
\hline Left-sided & $699(56)$ & $0(0)$ & N/A \\
\hline Acute & $312(25)$ & $0(0)$ & \\
\hline Chronic & $932(75)$ & $0(0)$ & \\
\hline Stroke & $32(3)$ & $1(0.05)$ & N/A \\
\hline \multicolumn{4}{|l|}{ Family history } \\
\hline Coronary artery disease & $21(2)$ & $1(0.05)$ & \\
\hline Peripheral artery disease & $7(1)$ & $0(0)$ & 0.835 \\
\hline \multicolumn{4}{|l|}{ Present medications } \\
\hline$\beta$-blockers & $247(20)$ & $0(0)$ & \\
\hline Calcium channel blockers & $152(12)$ & $0(0)$ & \\
\hline Hematinic & $41(3)$ & $42(2)$ & \\
\hline Immune Tonic & $31(3)$ & $35(2)$ & $<0.0001$ \\
\hline
\end{tabular}

The date was presented number (\%) for categorical characteristics and mean \pm SD for continuous data. Mann-Whitney U test was performed between groups for categorical data and unpaired t-test was performed between groups for continuous data. $\mathrm{P}<0.05$ was considered statistically significant. ${ }^{\mathrm{a}}<102 \mathrm{~cm}$ for men and $<88 \mathrm{~cm}$ for women were considered normal. ${ }^{\mathrm{b}} \mathrm{An}$ augmentation of fat in the hepatic cells. N/A, not applicable; SD, standard deviation.

Among these patients, 245 patients $<65$ years, images of 10 patients were indicated to be inadequate, 20 patients had cardiovascular diseases with 15 of whom had reported hypertension during measurements, 17 patients were obese and 27 patients had diabetes; therefore, these patients were excluded from the control population of the current study. A flowchart of the study is presented in Fig. 1.

A total of 1,244 patients were included in the test population. A total of 1,952 patients were included in the control population. There were no significant differences in height $(\mathrm{P}=0.056)$, sex $(\mathrm{P}=0.079)$, and family history for cardiac disease $(\mathrm{P}=0.835)$ but significant differences in blood serum creatinine $(\mathrm{P}=0.035)$ between the control and the test groups were observed. Both groups had the same numbers of smokers $(\mathrm{P}=0.066)$. The control population was younger than the test population $(\mathrm{P}<0.0001)$. Waist circumference $(\mathrm{P}<0.0001)$, patients who had consumed alcohol, $(\mathrm{P}<0.0001)$ and liver steatosis $(\mathrm{P}<0.0001)$ were higher in the test population compared with the control population. Unlike the control group, test group patients received a treatment of $\beta$-blockers or calcium channel blockers. Further demographic characteristics of enrolled patients are presented in Table I.

Routine measurements. Laboratory tests confirmed that the test population exhibited higher rates of obesity $(\mathrm{P}<0.0001)$, hypertension $(\mathrm{P}<0.0001)$, diabetes $(\mathrm{P}<0.0001)$, high heart rate $(\mathrm{P}<0.0001)$ and high BNP levels $(\mathrm{P}<0.0001)$ compared with the control population (Table II). 


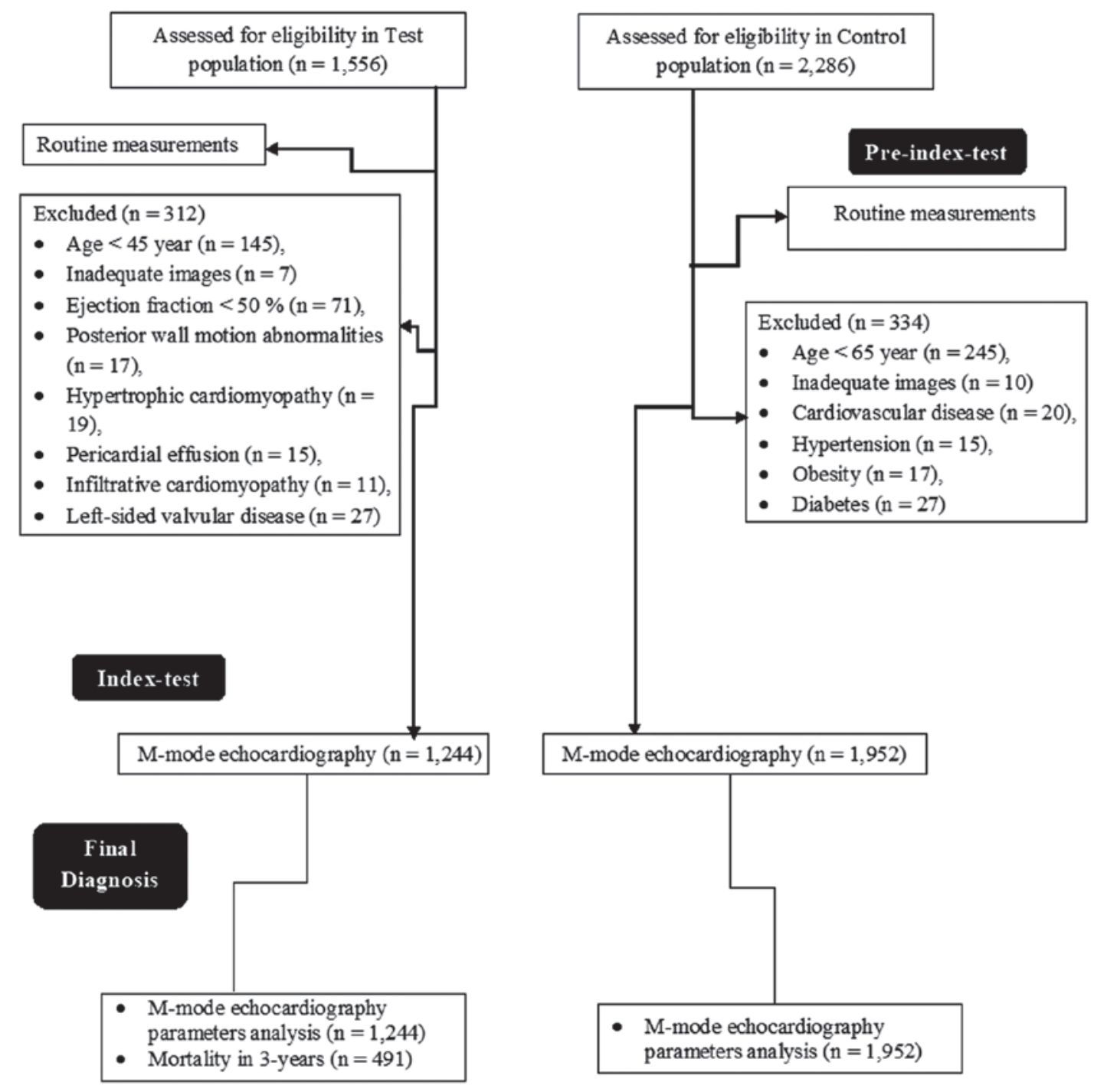

Figure 1. Flow diagram of the current study.

M-mode echocardiography evaluation. The median value of the 25th to 75th percentile DWS for the control population was 0.45 (range, $0.35-0.55$ ) and that of the test populations was 0.38 (range, 0.28-0.48). The distribution of DWS in the test populations of the current study was less compared with the control population (Fig. 2; $\mathrm{P}<0.0001$ ). Intra-observer and interobserver reliabilities were $0.0021 \pm 0.00051$ and $0.0031 \pm 0.00061$, respectively.

With respect to control population, the test population had the same left ventricular end-diastolic dimension $(\mathrm{P}=0.060)$ and the left ventricular end-diastolic volume $(\mathrm{P}=0.054)$. However, the test population had a higher left ventricular end-systolic dimension $(\mathrm{P}<0.0001)$, reduced $\mathrm{SF}(\mathrm{P}<0.0001)$, reduced posterior wall thickness at end-systole $(\mathrm{P}=0.007)$, reduced posterior wall thickness at end-diastole $(\mathrm{P}<0.0001)$, less DWS $(\mathrm{P}<0.0001)$, reduced mitral flow velocity $(\mathrm{P}<0.05)$, reduced myocardial wall motion velocity $(\mathrm{P}<0.05)$, increased Tei index $(\mathrm{P}<0.0001)$, increased left ventricular end-systolic volume $(\mathrm{P}<0.0001)$, and reduced ejection fraction $(\mathrm{P}<0.0001)$ compared with the control population (Table III).

Analysis of mortality. Patients from the test population who demonstrated the most severe M-mode echocardiography parameters, which were determined according to DWS, left ventricular end-diastolic volume, left ventricular end-systolic dimension, posterior wall thickness at end-systole and mitral flow velocity, died during a follow-up period of 3-years. Demographic characteristics and medical history did not appear to be associated with mortality (Table IV). A total of 491 patients from the test population died during the 3 -year follow-up period. In the test population, the numbers of patients with lower DWS $(<0.28)$ died within 3 -years of the follow-up and mortality rate was higher compared with the numbers of patients with higher $(\geq 0.28)$ DWS $(\mathrm{P}<0.0001$; Fig. 3).

\section{Discussion}

In a large comparative M-mode echocardiography study on patients with heart failure with preserved ejection fraction vs. normal (cardiac healthy) research subjects, DWS was indicated to be lower $(\mathrm{P}<0.0001)$ in the test population than in the cardiac healthy patients. Decreased DWS measurements were associated with worse outcomes, and within the test populations, patients with reduced DWS had a higher mortality rate 
Table II. Routine measurements.

\begin{tabular}{lcccc}
\hline & & \multicolumn{2}{c}{ Group } & Comparison between \\
\cline { 3 - 4 } Characteristics & Normal value $^{\mathrm{a}}$ & Test population & Control population & $\begin{array}{c}\text { Coups, P-value } \\
\text { groun }\end{array}$ \\
\hline Numbers of patients enrolled & N/A & 1,244 & 1,952 & $<$ \\
Weight, $\mathrm{kg}$ & $60-65$ & $75.15 \pm 7.12$ & $63.16 \pm 9.12$ & $<0.0001$ \\
BMI, $\mathrm{kg} / \mathrm{m}^{2}$ & $\leq 30$ & $30.87 \pm 3.15$ & $25.12 \pm 3.85$ & $<0.0001$ \\
Diastolic blood pressure, $\mathrm{mmHg}$ & 90 & $91 \pm 3$ & $85 \pm 4$ & $<0.0001$ \\
Systolic blood pressure, $\mathrm{mmHg}$ & 140 & $146 \pm 4$ & $131 \pm 8$ & $<0.0001$ \\
Heart rate, bpm & $60-100$ & $93 \pm 8$ & $86 \pm 4$ & $<0.0001$ \\
Random glucose, $\mathrm{mg} / \mathrm{dl}$ & $\leq 140$ & $171 \pm 9$ & $132 \pm 7$ & $<0.0001$ \\
The total cholesterol & $\leq 240$ & $261 \pm 9$ & $215 \pm 7$ & $<0.0001$ \\
LDL & $\leq 160$ & $171 \pm 8$ & $145 \pm 3$ & $<0.0001$ \\
HDL & $\geq 40$ & $33 \pm 3$ & $55 \pm 7$ & $<0.0001$ \\
HbA1c, $\%$ & $\leq 7$ & $8.2 \pm 0.5$ & $6.5 \pm 0.25$ & $<0.0001$ \\
BNP, pg/ml & $\leq 100$ & $115 \pm 8$ & $32 \pm 2$ & $<0.0001$ \\
\hline
\end{tabular}

${ }^{a}$ Normal value was based on a healthy Chinese person. Data were presented as mean \pm SD. Unpaired t-test was performed for statistical analysis. $\mathrm{P}<0.05$ was considered statistically significant. BMI, body mass index; BNP, brain natriuretic peptide; HDL, high-density lipoprotein; LDL, low-density lipoprotein; N/A, not applicable; SD, standard deviation.

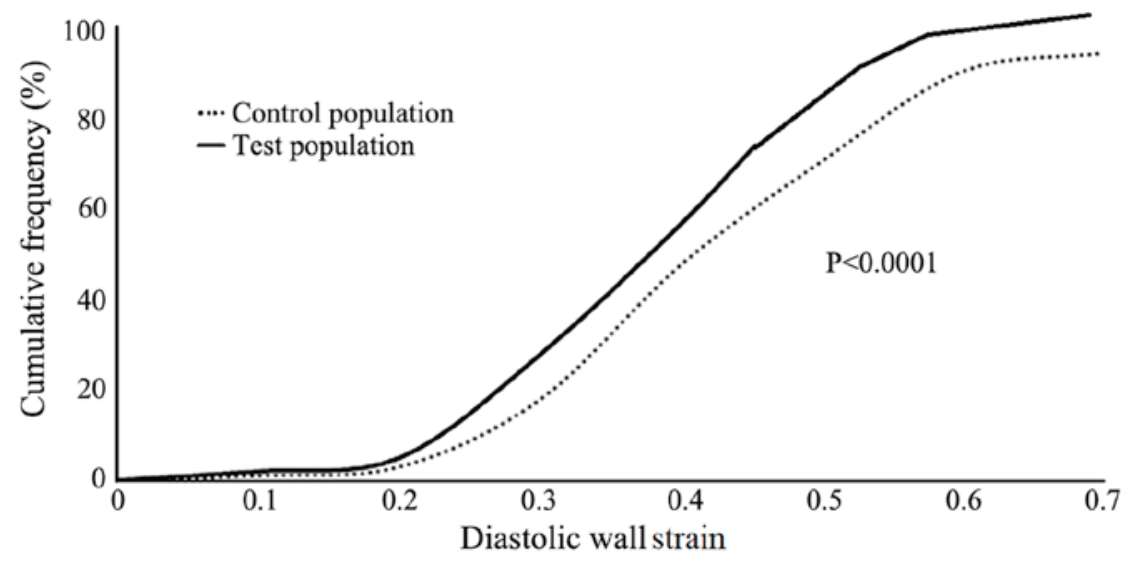

Figure 2. Cumulative frequency distribution of diastolic wall strain in the different study populations. Cardiac Ultrasonography was performed using M-mode echocardiography. The Fisher exact test was used for statistical analysis.

$(\mathrm{P}<0.0001)$. DWS is associated with left ventricle stiffness (17). Patients with heart failure and preserved ejection fraction exhibiting high diastolic stiffness, and lower DWS has been previously associated with higher mortality (7). DWS is easy to calculate and cost-effective (7). The DWS measurements of the present study were in line with previous studies $(7,17)$. In consideration of the results of the present study, DWS may be important M-mode echocardiography parameter for evaluating the risk of diastolic stiffness in patients with heart failure and preserved ejection fraction.

The present study demonstrated that the test population had high BNP, impaired left ventricular relaxation and lower myocardial wall motion velocity compared with the control population. Increased impaired left ventricular relaxation, raised BNP (18) and lower myocardial wall motion velocity results in the increased filling pressure of the left ventricle, in turn increasing its stiffness and poorer outcomes such as myocardial infraction (7). The results of the present study were consistent with previous studies $(7,19,20)$. However, these parameters are load dependent and intrinsic passive myocardial stiffness is not possible to predict (21). Furthermore, BNP results are also unreliable for predicting whether patients will have poor outcomes as BNP is also affected by renal function, obesity and pulmonary dysfunction (7,22). With respect to the diagnostic method adopted in the present study, M-mode echocardiography has provided valuable information, including DWS, E/e' ratio and mitral flow velocity, for the diagnosis of patients with heart failure and myocardial infarction.

Patients with heart failure and preserved ejection fraction (45-90 years) were younger than the control populations (45-90 years compared with 65-90 years, respectively; $\mathrm{P}<0.0001)$ but the left ventricular end-diastolic dimension $(\mathrm{P}=0.062)$ and the left ventricular end-diastolic volume 
Table III. Left ventricle structure and M-mode echocardiography parameters.

\begin{tabular}{|c|c|c|c|}
\hline \multirow[b]{2}{*}{ Characteristics } & \multicolumn{2}{|c|}{ Group } & \multirow{2}{*}{$\begin{array}{c}\text { Comparison between } \\
\text { groups, P-value }\end{array}$} \\
\hline & Test population & Control population & \\
\hline Numbers of patients enrolled & 1,244 & 1,952 & $\mathrm{~N} / \mathrm{A}$ \\
\hline LVIDs, mm & $30.81 \pm 5.45^{\mathrm{a}}$ & $29.92 \pm 4.51$ & $<0.0001$ \\
\hline LVIDd, mm & $46.92 \pm 5.45$ & $47.46 \pm 9.15$ & 0.060 \\
\hline Shortening fraction, $\%$ & $31.23 \pm 3.41^{\mathrm{a}}$ & $35.45 \pm 5.41$ & $<0.0001$ \\
\hline Posterior wall thickness at end-systole & $10.01 \pm 2.01^{\mathrm{a}}$ & $10.21 \pm 2.11$ & 0.007 \\
\hline Posterior wall thickness at end-diastole & $14.01 \pm 1.91^{\mathrm{a}}$ & $15.12 \pm 2.92$ & $<0.0001$ \\
\hline DWS & $0.30 \pm 0.07^{\mathrm{a}}$ & $0.34 \pm 0.05$ & $<0.0001$ \\
\hline Mitral flow velocity & $1.78 \pm 2.45^{\mathrm{a}}$ & $2.15 \pm 6.21$ & 0.040 \\
\hline Myocardial wall motion velocity & $6.09 \pm 1.95^{\mathrm{a}}$ & $6.28 \pm 2.11$ & 0.004 \\
\hline Tei index & $0.39 \pm 0.016^{\mathrm{a}}$ & $0.38 \pm 0.015$ & $<0.0001$ \\
\hline $\mathrm{EDV}, \mathrm{ml}$ & $162.25 \pm 5.47$ & $161.51 \pm 12.81$ & 0.053 \\
\hline $\mathrm{ESV}, \mathrm{ml}$ & $81.15 \pm 4.47^{\mathrm{a}}$ & $80.12 \pm 4.14$ & $<0.0001$ \\
\hline$\% \mathrm{EF}$ & $59.12 \pm 2.91^{\mathrm{a}}$ & $63.35 \pm 3.12$ & $<0.0001$ \\
\hline
\end{tabular}

Data were presented as mean $\pm \mathrm{SD}$. Unpaired t-test was performed for statistical analysis. $\mathrm{P}<0.05$ was considered statistically significant.

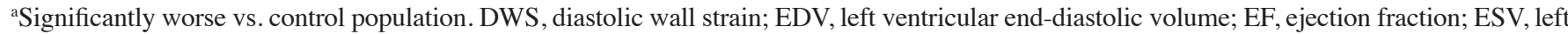
ventricular end-systolic volume; LVIDd, end-diastolic dimension; LVIDs, end-systolic dimension; N/A, not applicable; SD, standard deviation.

Table IV. Multiple regression analysis for mortality in the test population during follow-up of 3-years.

\begin{tabular}{ll}
\hline Characteristics & $\begin{array}{c}\text { P-value for the } \\
\text { analysis of parameter }\end{array}$ \\
\hline LVIDs, mm & $0.001^{\mathrm{a}}$ \\
LVIDd, mm & $0.002^{\mathrm{a}}$ \\
Shortening fraction, \% & $0.015^{\mathrm{a}}$ \\
Posterior wall thickness at end-systole & $0.025^{\mathrm{a}}$ \\
Posterior wall thickness at end-diastole & $0.026^{\mathrm{a}}$ \\
DWS & $0.001^{\mathrm{a}}$ \\
The mitral flow velocity & $0.002^{\mathrm{a}}$ \\
The myocardial wall motion velocity & $0.021^{\mathrm{a}}$ \\
Tei index & $0.031^{\mathrm{a}}$ \\
EDV, ml & $0.041^{\mathrm{a}}$ \\
ESV, ml & $0.032^{\mathrm{a}}$ \\
$\%$ EF & $0.021^{\mathrm{a}}$ \\
Age, years & 0.12 \\
Sex & 0.15 \\
Body mass index, kg/m ${ }^{2}$ & 0.17 \\
Smoking & 0.55 \\
Family history & 0.69 \\
Brain natriuretic peptide, pg/ml & 0.052 \\
Hiabetes & 0.062 \\
\hline Pspertension & 0.075 \\
\hline
\end{tabular}

$\mathrm{P}<0.05$ was considered statistically significant. ${ }^{\text {a Significant parameter }}$ associated with mortality. Data of patients who survived were considered as a reference. DWS, diastolic wall strain; EDV, left ventricular end-diastolic volume; EF, ejection fraction; ESV, left ventricular end-systolic volume; LVIDd, left ventricular end-diastolic dimension; LVIDs, left ventricular end-systolic dimension.
$(\mathrm{P}=0.054)$ were the same between the groups. The present study aimed to assess if left ventricular end-diastolic dimension and the left ventricular end-diastolic volume were successfully predicted by M-mode echocardiography. If the present study had enrolled patients of equal age for both groups, it is possible that the results could be different for left ventricular end-diastolic dimension and the left ventricular end-diastolic volume between for both groups. In this condition, the study should perform multivariate analysis to evaluate the effect of age on left ventricular end-diastolic dimension and the left ventricular end-diastolic volume considering the control group as the reference standard. However, in the present study, logistic regression analysis was not possible, using the control group as a reference standard because height, waist circumference, present medication and other demographic parameters varied between the control and test populations. Therefore, the present study enrolled patients (age range, 45-90 years) with heart failure and preserved ejection fraction in the test group, and 65-90 years old patients without known cardiovascular disease in the control population. Additionally, the left ventricular end-diastolic dimension and volume declines with age (14,23). Left ventricular end-diastolic dimension and left ventricular end-diastolic volume are successfully evaluated by the present M-mode echocardiographic study. There is a requirement for further tailored made studies investigating the relationship between M-mode echocardiography and demographical characteristics of patients with heart failure and preserved ejection fraction, on adverse outcomes.

The present study used comparative M-mode echocardiography to investigate the outcome in patients with heart failure and preserved ejection fraction. Unlike M-mode echocardiography, cardiac magnetic resonance (CMR) can acquire images in any plane and/or any long axis, and diastolic stiffness 


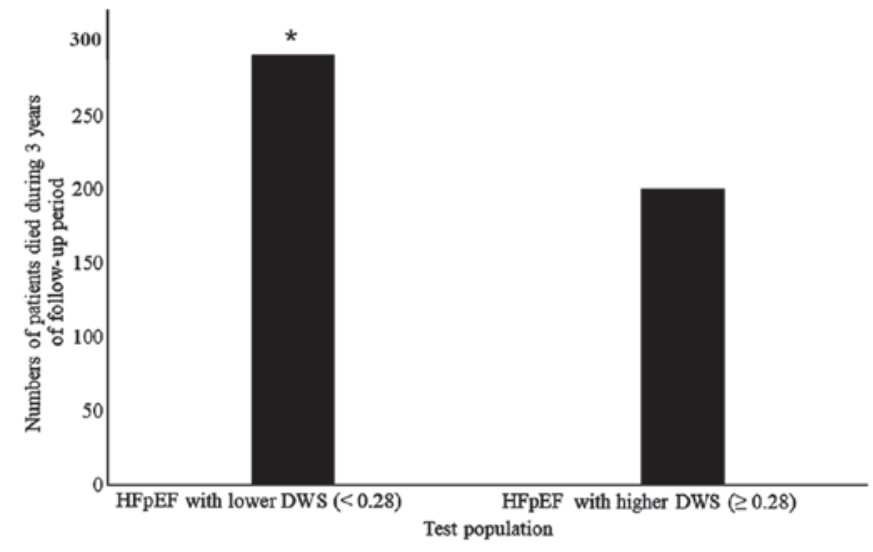

Figure 3. Mortality of the test population at the 3-year of follow-up. The Fisher exact test was used for statistical analysis. A DWS of 0.28 was selected as a lower limit because it was the 25th percentile of the test populations. DWS, Diastolic wall strain; HFpEF, Heart failure patients with preserved ejection fraction. ${ }^{*} \mathrm{P}<0.0001$.

parameters have been found to be similar to those of M-mode echocardiography $(1,24)$. However, CMR has limited facilities in China and T1 CMR mapping data in patients with heart failure and preserved ejection fraction are still limited (25). Therefore, the present study adopted traditional M-mode echocardiography to perform the study.

During the present study, following a 3-year follow-up period, $\sim 40 \%$ of patients died. This is higher than demonstrated in previous studies $(2,7,19,20)$. It is well-established that systolic regional thickening abnormalities in patients with heart failure patients and preserved ejection fraction accompany significant ischemia, but systolic regional thickening cannot precisely identify ischemic territories (26). Therefore, the finding that patients with lower systolic regional thickening have a higher mortality rate may be an important finding in itself.

In the present study, the DWS values were $0.30 \pm 0.007$ in the test population, but all test population patients were hypertensive, diabetic and possibly under medication. The echocardiogram profile appeared similar to the normal values for the test population. However, since significant differences were observed in all echocardiogram parameters in the test population compared with the control group $(\mathrm{P}<0.05)$. Therefore, there is the possibility of the false positive errors ( $\alpha$-errors) for echocardiogram parameters of the present study. The present study performed multiple regression analysis to predict mortality. Multiple regression analysis showed that the left ventricular end-systolic and end-diastolic dimension, SF, posterior wall thickness at end-systole and at end-diastole, DWS, the mitral flow velocity, the myocardial wall motion velocity and the other abnormal echocardiogram parameters of the test population were associated with a higher mortality.

There are a number of limitations of the present study. DWS was found to be significantly lower in control patients. The reason behind these false low values of DWS in the normal subjects is not clear. The effect of the different types of heart failure on M-mode echocardiography parameters were not evaluated. M-mode echocardiography parameters were not compared with CMR T1 images. Although none of the patients in the present study exhibited abnormalities of regional wall motion, M-mode echocardiography parameters do not provide reliable results in such cases (27), instead, speckle tracking echocardiography should be performed in such patients.

In conclusion, the majority of echo parameters between the two groups were statistically different. Each of these parameters were found to be sufficient to indicate a higher mortality rate among patients with heart failure and preserved ejection fraction, similar to that of DWS. Poor outcomes in Chinese patients with heart failure patients and preserved ejection fraction can be predicted using DWS measurements and further M-mode echocardiography parameters. Echocardiography is a useful tool for diagnosing the clinically important dilemma of heart failure with preserved ejection fraction.

\section{Acknowledgements}

Not applicable.

\section{Funding}

No funding was received.

\section{Availability of data and materials}

The datasets used and/or analyzed during the current study are available from the corresponding author on reasonable request.

\section{Authors' contributions}

All authors had read and approved submission for publication. XL was a project administrator who contributed to the conceptualization and literature review of the study. XM contributed to the formal analysis, data curation, software analysis, and drafted and edited the manuscript for intellectual content. The authors agree to be accountable for all aspects of work ensuring integrity and accuracy.

\section{Ethics approval and consent to participate}

The study had been registered in the Research registry (www.researchregistry.com), UID No. researchregistry4465 (https://www.researchregistry.com/register-now\#home/regis trationdetails/5bc043c4e8a7130a3e730a64/) dated 1 August 2012. The protocol (ADH/HUM/CL/5/15 dated 25 July 2012) had been granted by the Affiliated Dongfeng Hospital review board. The study adhered to the laws of China, standards for the reporting of diagnostic accuracy studies (http://www. equator-network.org/reporting-guidelines/stard/) guidelines, the strengthening the reporting of observational studies in epidemiology (STROBE) statement, and the Declaration of Helsinki (V2008). All patients had signed an informed consent form regarding radiology and pathology.

\section{Patient consent for publication}

Not applicable. 


\section{Competing interests}

The authors declare that they have no competing interests.

\section{References}

1. Paulus WJ, Tschope C, Sanderson JE, Rusconi C,Flachskampf FA, Rademakers FE, Marino P, Smiseth OA, De Keulenaer G, Leite-Moreira AF, et al: How to diagnose diastolic heart failure: A consensus statement on the diagnosis of heart failure with normal left ventricular ejection fraction by the Heart Failure and Echocardiography Associations of the European Society of Cardiology. Eur Heart J 28: 2539-2550, 2007.

2. Yap J, Sim D, Lim CP, Chia SY, Go YY, Jaufeerally FR, Sim LL, Liew R and Ching CK: Predictors of two-year mortality in Asian patients with heart failure and preserved ejection fraction. Int J Cardiol 183: 33-38, 2015.

3. Lee R, Chan SP, Chan YH, Wong J, Lau D and Ng K: Impact of race on morbidity and mortality in patients with congestive heart failure: A study of the multiracial population in Singapore. Int J Cardiol 134: 422-425, 2009.

4. West R, Liang L, Fonarow GC, Kociol R, Mills RM, O'Connor CM and Hernandez AF: Characterization of heart failure patients with preserved ejection fraction: A comparison between ADHERE-US registry and ADHERE-International registry. Eur J Heart Fail 13: 945-952, 2011.

5. King A: Heart failure: Registry data highlight global differences in care for HF-PEF. Nat Rev Cardiol 8: 482, 2011.

6. Ferrari R, Bohm M, Cleland JG, Paulus WJ, Pieske B, Rapezzi C and Tavazzi L: Heart failure with preserved ejection fraction: Uncertainties and dilemmas. Eur J Heart Fail 17: 665-671, 2015.

7. Ohtani T, Mohammed SF, Yamamoto K, Dunlay SM, Weston SA, Sakata Y, Rodeheffer RJ, Roger VL and Redfield MM: Diastolic stiffness as assessed by diastolic wall strain is associated with adverse remodelling and poor outcomes in heart failure with preserved ejection fraction. Eur Heart J 33: 1742-1749, 2012.

8. Takeda Y, Sakata Y, Higashimori M, Mano T, Nishio M, Ohtani T, Hori M, Masuyama T, Kaneko M and Yamamoto K: Noninvasive assessment of wall distensibility with the evaluation of diastolic epicardial movement. J Card Fail 15: 68-77, 2009.

9. Omar AM, Bansal $M$ and Sengupta PP: Advances in Echocardiographic imaging in heart failure with reduced and preserved ejection fraction. Circ Res 119: 357-374, 2016.

10. Tribouilloy C, Rusinaru D, Mahjoub H, Goissen T, Lévy F and Peltier M: Impact of echocardiography in patients hospitalized for heart failure: A prospective observational study. Arch Cardiovasc Dis 101: 465-473, 2008.

11. Kanaya AM, Kandula NR, Ewing SK, Herrington D, Liu K, Blaha MJ, Srivastava S, Dave SS and Budoff MJ: Comparing coronary artery calcium among U.S. South Asians with four racial/ethnic groups: The MASALA and MESA studies. Atherosclerosis 234: 102-107, 2014.

12. Newby DE, Williams MC, Flapan AD, Forbes JF, Hargreaves AD, Leslie SJ, Lewis SC, McKillop G, McLean S, Reid JH, et al: Role of multidetector computed tomography in the diagnosis and management of patients attending the rapid access chest pain clinic, The Scottish computed tomography of the heart (SCOT-HEART) trial: Study protocol for randomized controlled trial. Trials 13: 184, 2012.

13. Hulsmann M, Berger R, Mortl D, Gore O, Meyer B and Pacher R: Incidence of normal values of natriuretic peptides in patients with chronic heart failure and impact on survival: A direct comparison of $\mathrm{N}$-terminal atrial natriuretic peptide, $\mathrm{N}$-terminal brain natriuretic peptide and brain natriuretic peptide. Eur J Heart Fail 7: 552-556, 2005.
14. Suzue M, Mori K, Inoue M, Hayabuchi Y, Nakagawa R and Kagami S: Developmental changes in the left ventricular diastolic wall strain on M-mode echocardiography. J Echocardiogr 12: 98-105, 2014

15. Blanchard DG, Malouf PJ, Gurudevan SV, Auger WR, Madani MM, Thistlethwaite P, Waltman TJ, Daniels LB, Raisinghani AB and DeMaria AN: Utility of right ventricular Tei index in the noninvasive evaluation of chronic thromboembolic pulmonary hypertension before and after pulmonary thromboendarterectomy. JACC Cardiovasc Imaging 2: 143-169, 2009.

16. Lurz P, Eitel I, Adam J, Steiner J, Grothoff M, Desch S, Fuernau G, de Waha S, Sareban M, Luecke C, et al: Diagnostic performance of CMR imaging compared with EMB in patients with suspected myocarditis. JACC Cardiovasc Imaging 5: 513-524, 2012.

17. Hay I, Rich J, Ferber P, Burkhoff D and Maurer MS: Role of impaired myocardial relaxation in the production of elevated left ventricular filling pressure. Am J Physiol Heart Circ Physiol 288: H1203-H1208, 2005.

18. Grewal J, McKelvie R, Lonn E, Tait P, Carlsson J, Gianni M, Jarnert $\mathrm{C}$ and Persson H: BNP and NT-proBNP predict echocardiographic severity of diastolic dysfunction. Eur J Heart Fail 10: 252-259, 2008.

19. Zile MR, Gaasch WH, Anand IS, Haass M, Little WC, Miller AB, Lopez-Sendon J, Teerlink JR, White M, McMurray JJ, et al: Mode of death in patients with heart failure and a preserved ejection fraction: Results from the Irbesartan in Heart Failure with Preserved Ejection Fraction Study (I-Preserve) trial. Circulation 121: 1393-1405, 2010.

20. Lam CS, Roger VL, Rodeheffer RJ, Borlaug BA, Enders FT and Redfield MM: Pulmonary hypertension in heart failure with preserved ejection fraction: A community-based study. J Am Coll Cardiol 53: 1119-1126, 2009.

21. Tschope $C$ and Paulus WJ: Is echocardiographic evaluation of diastolic function useful in determining clinical care? Doppler echocardiography yields dubious estimates of left ventricular diastolic pressures. Circulation 120: 810-820, 2009.

22. Borlaug BA and Paulus WJ: Heart failure with preserved ejection fraction: Pathophysiology, diagnosis and treatment. Eur Heart J 32: 670-679, 2011

23. CainPA, Ahl R,HedstromE,Ugander M,Allansdotter-Johnsson A, Friberg $\mathrm{P}$ and Arheden H: Age and sex-specific normal values of left ventricular mass, volume and function for gradient echo magnetic resonance imaging: A cross-sectional study. BMC Med Imaging 9: 2, 2009.

24. Yang XS and Sun JP: Advances in diastolic heart failure. World J Cardiol 2: 58-63, 2010.

25. Rommel KP, Lucke C and Lurz P: Diagnostic and prognostic value of CMR T1-mapping in patients with heart failure and preserved ejection fraction. Rev Esp Cardiol (Engl Ed) 70: 848-855, 2017.

26. Tsaftaris SA, Zhou X, Tang R, Li D and Dharmakumar R: Detecting myocardial ischemia at rest with cardiac phase-resolved blood oxygen level-dependent cardiovascular magnetic resonance. Circ Cardiovasc Imaging 6: 311-319, 2013.

27. Omar AM, Vallabhajosyula S and Sengupta PP: Left ventricular twist and torsion: Research observations and clinical applications. Circ Cardiovasc Imaging 8: e003029, 2015. 